\title{
Design and Development of Lower Limb Chair Exercise Support System with Depth Sensor
}

\author{
${ }^{1}$ Toshiya Watanabe, ${ }^{2}$ Naohiro Ohtsuka, ${ }^{3}$ Susumu Shibusawa, ${ }^{4}$ Masaru Kamada and \\ ${ }^{5}$ Tatsuhiro Yonekura \\ ${ }^{1}$ Graduate School of Science and Engineering, Ibaraki University, Hitachi, Japan; \\ ${ }^{2}$ East Japan Institute of Technology Co., Ltd; \\ ${ }^{3,4,5}$ School of Engineering, Ibaraki University, Hitachi, Japan; \\ 112nd307a@hcs.ibaraki.ac.jp; ${ }^{3}$ sibusawa@mx.ibaraki.ac.jp; ${ }^{4}$ m.kamada@mx.ibaraki.ac.jp; \\ ${ }^{5}$ yone@mx.ibaraki.ac.jp
}

\begin{abstract}
Sustaining lower limb functionality is extremely important in the preventative care of the elderly. Chair exercise, in which the exerciser sits on an ordinary chair, offers a way for seniors with little physical strength to exercise without a great deal of effort. Meanwhile, Microsoft's Kinect sensor that is capable of detecting human motion without the subject having to wear any kind of a special marker are becoming widely available. Exploiting this new sensor technology, this paper describes the design and development of a prototype lower limb chair exercise support system. The system supports five different chair exercises designed to strengthen the lower limbs, recognizes and evaluates exercises based on 3D position data and joint angles for each joint obtained from the Kinect sensor. The system illustrates how to do the exercises by voice instructions and model images, and superimposes the muscles used onto an image of the exerciser in real time. The system also provides exercise assessment results and advice by voice and text. In a series of trials involving seven elderly subjects in their late 70 s and early 80 s, an overall average recognition rate of $89 \%$ was obtained for the five exercises. Feedback was obtained through a questionnaire given to subjects ranging in age from 50 to 65 , which highlighted a number of issues that should be addressed to improve the effectiveness of the system.
\end{abstract}

Keywords: chair exercise; lower limb; elderly population; depth sensor; exercise system design; preventative care.

\section{Introduction}

Japan is rapidly becoming a super-aged society with over $25 \%$ of the population aged 65 or older, and the number of elderly requiring long-term care is rapidly increasing [1]. Good preventative care that enables as many people as possible to live independently in sound health is thus becoming increasingly important. The goals of preventative long-term care are to stave off the need for long-term care as long as possible, to prevent one's condition from deteriorating in cases where care is already required, and to enhance peoples' quality of life by improving their mental and physical fitness [2]. Preventative care is typically overseen by a physical therapist or other professional, but obviously there are not enough healthcare professionals to deal with the entire elderly cohort in the population. This is where support systems come in that can effectively stave off or prevent nursing care among seniors. 
For the purposes of preventative care, sustaining lower limb functionality is critically important for enabling people to keep their balance, to continue walking, and to prevent falls that often lead to bedridden debilitation, and considerable interest has focused on chair exercise as an excellent way to strengthen the lower limbs. Exercise done from a sitting position in a chair is ideally suited for older people who lack stamina, because it takes the burden off of their ankles and knee joints so they can strengthen leg muscles with relatively little effort. Not surprisingly, chair exercise is used extensively in many nursing homes and elder care facilities.

Preventative care solutions and rehabilitation have become increasingly important as society ages, and have prompted a surge of research interest into systems that might assist rehabilitation and longterm preventative care. In the work of Matsukuma et al. on stand-up rehabilitation for older people [3], these authors created a game out of the repetitive stand-up and sit-down exercise that is widely prescribed to rehab patients, and they found that the addition of this entertainment aspect markedly improved the persistence and enthusiasm of patients. Hashimoto et al. [4] developed a rehabilitation site [5] that patients can access over the Internet from the comfort of their own homes enabling them to pursue rehabilitation at their own pace.

Meanwhile, Microsoft's Kinect has become readily available for tracking user movement and posture. Kinect features an RGB camera and a depth sensor that captures user movements and 3D positions for up to 20 different joint points without the need of special markers or putting a sensor on the body. Moreover, because Kinect is a compact device engineered for use in affordable games, it is already being widely deployed and used in ordinary homes, and is expected to see many new applications in medical, disability support, and other healthcare-related areas [6-10].

In this paper, we present the design methodology, development and evaluation of a lower limb chair exercise support system with the Kinect depth sensor. The system supports five different chair exercises for strengthening the lower limbs, and each exercise is recognized and evaluated using 3D positioning and joint angle data captured by Kinect. The user is first shown how to do the exercises by voice instructions and model images, then the user's use of muscles in performing the exercise is superimposed on the image in real time. The system also provides assessment results and advice by voice and text. Toe and heel exercises are implemented by estimating the toe position from Kinect depth images.

The rest of the paper is organized as follows: In Chapter 2, we review other relevant research, and Chapter 3 details the design of the Kinect-based chair exercise support system. A prototype chair exercise support system is described in Chapter 4. In Chapter 5, we evaluate the prototype system through a series of trials and consider the implications of the trial results, and Chapter 6 provides a brief recap of the study.

\section{Related Work}

Microsoft Kinect is a compact affordable device with built-in RGB camera, depth sensor, and other features designed specifically for use in game consoles. The ability of Kinect to capture 3D positions and movement without the need of special markers or putting a sensor on the body has attracted enormous interest among researchers for developing medical and disability support systems and devices. For example, Mentiplay et al. studied the reliability and effectiveness of Kinect for evaluating static foot postures [6], and found that Kinect is much more reliable than prevailing visual indicators for assessing static foot posture, and yielded results that are approximately equivalent to those derived from 3D motion analysis. Metcalf et al. examined markerless motion capture and measurement of hand kinematics [7], and proposed using Kinect for the measurement and analysis of 
Toshiya Watanabe, Naohiro Ohtsuka, Susumu Shibusawa, Masaru Kamada and Tatsuhiro Yonekura; Design and Development of Lower Limb Chair Exercise Support System with Depth Sensor, Transactions on Networks and Communications, Volume 3 No 4, August (2015); pp: 30-44

complex and hard-to-measure finger movements. These authors also emphasized that Kinect is far easier to apply in home-bound upper limb rehabilitation schemes than marker-based motion capture.

Obdrzalek et al. sought to evaluate the accuracy of pose measurements using Kinect in the context of exercise for an elderly population [8]. Meanwhile, Erazo et al. developed a rehabilitation system using Kinect based on the magic mirror game for people with upper limb impairments, and evaluated the effectiveness of the system [9]. Watanabe et al. showed the design of the lower limb chair exercise support system with Kinect, developed a prototype system, and conducted trials for elderly subjects $[11,12]$.

In recent years, we have seen growing interest in serious game or gamification, where game thinking and game mechanics are used in non-game contexts such as to improve user engagement in learning and training $[13,14]$. In the work on stand-up rehabilitation, Matsukuma et al. developed a game that improves patient motivation and persistence to do the rehabilitation exercise which is strongly recommended [3]. Another study in this vein is a 3D virtual rehabilitation game called Dance2Rehab3D by Bruckheimer et al. [15]. This upper limb rehabilitation support system uses joint angle data provided by Kinect in an interactive 3D environment based on an aquarium as a motif. Experimental results indicate that this approach is effective at strengthening upper limbs and reducing fatigue of stroke patients.

Asakura et al. have developed a shoulder rehabilitation support system that combines image-based 3D pose measurement data with myoelectric sensing data, and emphasizes presentation of information on the user's current condition and what the user might do to improve his or her fitness [16]. Pei et al. published a study on a robotic lower limb rehabilitation musculo-skeletal model [17] featuring a lower limb rehabilitation scheme that exploits robotic capability to track complex orbits, and compared the rehabilitation efficiency of their approach with existing methods. Fasola et al. [18] used the socially assistive human-robot interaction for older adult physical exercise, and showed that elderly users have a strong preference for the relational robot in terms of enjoyableness.

Igarashi et al. devised a wearable light-emitting sensor suit as a way of supporting lower-limb motion perception by visual means [19]. By measuring myoelectric potential and joint angles associated with motion, these authors are able to show muscle activity in real time for any location site of lower limb muscle activity on the body surface.

Several commercial rehabilitation systems based on Kinect have been developed [20-22]. However, these systems show neither the design methodology on support systems nor the quantitative evaluation, and we are not aware of any comprehensive study for such systems. A rehabilitation system to publish a part of method acquiring patient motion does not show the quantitative evaluation on lower limb chair exercise [23-26].

We show the design methodology, development and evaluation of a lower limb chair exercise support system with the Kinect depth sensor in this paper.

\section{System Design}

\subsection{Exercise Support System Requirements}

Desirable exercise support system has functions to $[9,11]$ :

(1) provide exercise guidance using images, text, and voice instructions,

(2) verify a user's posture and form while exercising, 
(3) evaluate the exerciser's efforts and offer advice in the form of video, text, and speech,

(4) motivate users and encourage users to continue exercising,

(5) save exercise records,

(6) enable the therapist to modify or tailor the application system to the exerciser's fitness level, and

(7) include game elements to motivate and encourage users.

Requirements (1) and (2) show images of the exerciser together with instructions on how the exercise is done correctly, so this makes the user aware of what must be done to adhere to the proper form of the exercise. Video and audio showing the effectiveness of the exercises in everyday life situations gives users a better understanding of the specific benefits of the exercises.

Requirements (3), (4), and (7) help users understand the benefits of the exercise and improve their motivation by evaluating their form and providing instant feedback. Encouraging and motivating users to stick with the exercise program is extremely important. Negative expressions might have the contrary effect of discouraging elderly users, so negative feedback is avoided in an effort to bring users back to the program and persist with the exercise.

\subsection{System Overview}

In this work, a prototype chair exercise support system was developed that employs a Kinect sensor to capture 3D position data of the user that is used to assess and critique the form of the user performing the exercise. Figure 1 shows a schematic overview of the system. As one can see from the figure, the Kinect sensor is positioned directly in front of the user who is seated in a chair, and video data of the user captured by Kinect is sent to a computer for processing. Audio and text feedback are provided in real time that accompany video images and exercise data that are displayed on a screen or monitor.

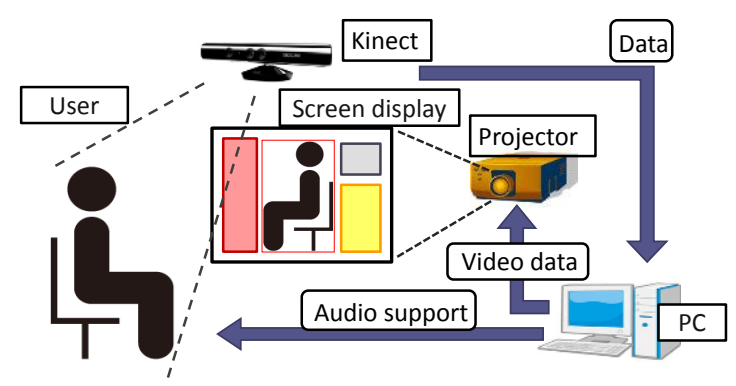

Figure 1: System overview
Table 1: System functions

\begin{tabular}{|c|l|l|}
\hline & \multicolumn{1}{|c|}{ Function } & \multicolumn{1}{c|}{ Description } \\
\hline (1) & $\begin{array}{l}\text { Display images of the } \\
\text { exerciser }\end{array}$ & Display RGB image captured by Kinect. \\
\hline (2) & Display muscles used & $\begin{array}{l}\text { Display image of muscles used as the user } \\
\text { exercises superimposed on the RGB image. }\end{array}$ \\
\hline$(3)$ & $\begin{array}{l}\text { Display effects of } \\
\text { exercises }\end{array}$ & $\begin{array}{l}\text { Visually display scenes illustrating effects of } \\
\text { exercise in everyday life. }\end{array}$ \\
\hline$(4)$ & Evaluate exercises & $\begin{array}{l}\text { Evaluate exercise on a 4-point scale: excellent, } \\
\text { good, fair, or poor. Evaluation results are presented } \\
\text { in voice and text for each exercise. }\end{array}$ \\
\hline$(5)$ & $\begin{array}{l}\text { Save exercise } \\
\text { records }\end{array}$ & $\begin{array}{l}\text { Record support system use data, joint angle and } \\
\text { position data for each exercise, evaluation data. }\end{array}$ \\
\hline
\end{tabular}

\subsection{Exercise System Functions}

Table 1 lists the primary functions of the system. As outlined in Section 3.1, the basic functions of the exercise support system are (1) to display images of the exerciser, (2) to explain in images and text exactly how to do the exercises, (3) to highlight the beneficial effects of the exercises, (4) to display evaluation results of the exercises in speech and text, and (5) to record and store data for each exercise.

\subsection{Exercise Recognition Method}

The system supports five different exercises for strengthening the lower limbs that are done while sitting in a chair: toe lifts, heel lifts, knee extensions, thigh lifts, and leg-open exercises. Just two criteria are used to identify or recognize these different exercises: 3D position data of various joints captured by Kinect, and joint angles derived from the 3D Kinect data. For the purposes of exercise recognition, 
Toshiya Watanabe, Naohiro Ohtsuka, Susumu Shibusawa, Masaru Kamada and Tatsuhiro Yonekura; Design and Development of Lower Limb Chair Exercise Support System with Depth Sensor, Transactions on Networks and Communications, Volume 3 No 4, August (2015); pp: 30-44

here we employ the following abbreviations for joints: toe $=\mathrm{To}$, ankle $=\mathrm{A}$, knee $=\mathrm{K}$, hips $=\mathrm{Hi}$, hip on open leg side $=m_{-} \mathrm{Hi}$, and hip on stationary leg side $=\mathrm{s}_{-} \mathrm{Hi}$.

\subsubsection{Toe-Lift Exercise}

The toe-lift exercise is done by raising the toes while leaving the heels planted firmly on the ground. If the toe position estimated from the depth image is represented by To and the ankle joint position derived from Kinect is represented by $A$, the toe-lift exercise is recognized using the ankle joint angle a between a straight line connecting A-To and a horizontal line and the toe height yT. The ankle joint angle and toe height used to recognize the toe-lift exercise are illustrated in Figure 2.

Changes in the ankle joint angle and toe position when doing the toe-lift exercise are shown in Figure 3. As one can see in the figure, the value of the ankle joint angle decreases as the toe position increases when doing the exercise.

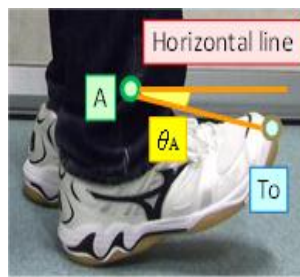

(a) Joint angle

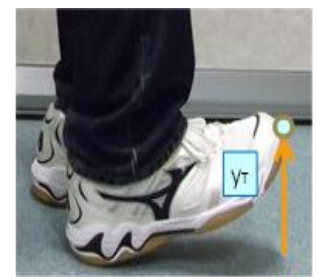

(b) Toe position

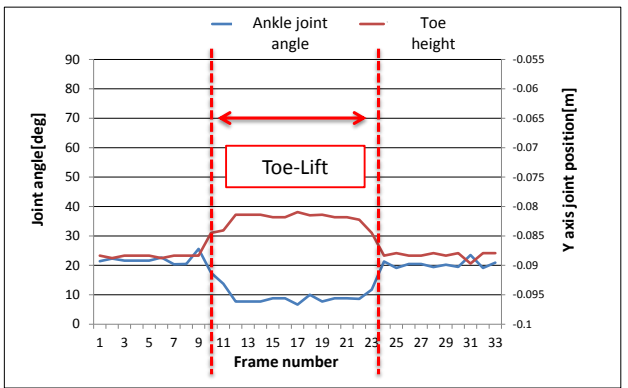

Figure 3: Angle and position change during the toe-lift exercise.

\subsubsection{Heel-Lift Exercise}

The heel-lift exercise is done by placing weight on the balls of the feet and raising the heels. If the toe position estimated from the depth image is represented by To and the ankle joint position derived from Kinect is represented by $\mathrm{A}$, the heel-lift exercise is recognized using the ankle joint angle $\theta_{\mathrm{A}}$ between a straight line connecting A-To and a horizontal line and the ankle height $y_{\mathrm{K}}$. The joint angle and joint position used to recognize the heel-lift exercise are illustrated in Figure 4.

Changes in the ankle joint angle and knee position when doing the heel-lift exercise are shown in Figure 5. One can see in Figure 5(a) that the ankle joint angle increases as you do the heel-lift exercise. While the ankle height does not change that much, the knees are elevated to a moderate degree, as shown in Figure $5(b)$. The system recognizes the heel-lift exercise by focusing on the changing ankle joint angle $\theta_{\mathrm{A}}$ and knee joint height $y_{\mathrm{K}}$ of the exerciser, and determines that the exercise is being performed when these values satisfy the relevant thresholds.

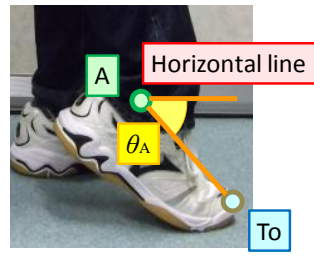

(a) Joint angle

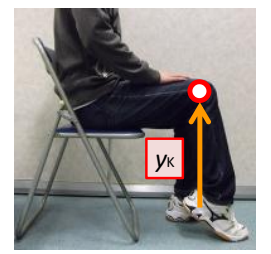

(b) Joint position
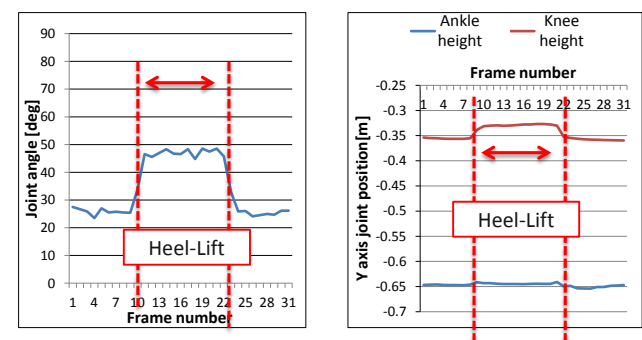

Figure 5: Changing joint angles and positions when doing the heel-lift exercise
Figure 4: Joint angle and position used in the heel-lift exercise 


\subsubsection{Knee-Extension Exercise}

The knee-extension exercise is done by rising one leg at a time and extending or straightening the knee. Where $\mathrm{Hi}, \mathrm{K}$, and $\mathrm{A}$ represent hip, knee, and ankle, respectively, the knee-extension exercise is recognized using the knee joint angle 跃 formed from these three joints and the ankle height $\mathrm{yA}$. The joint angle and joint positions used to recognize the knee-extension exercise are illustrated in Figure 6.

Changes in the knee joint angles and ankle positions when doing the knee-extension exercise are shown in Figure 7. One will note in the figure that the value of the knee joint angle increases as the ankle position is elevated when doing the knee-extension exercise. Thus, the system recognizes the

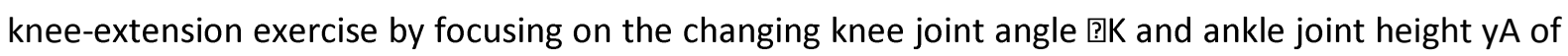
the exerciser, and determines that the exercise is being performed when these value satisfy the relevant thresholds

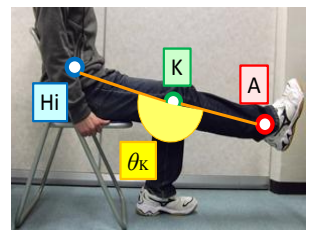

(a) Joint angles

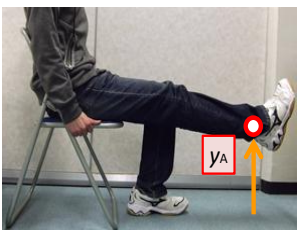

(b) Joint positions

Figure 6: Joint angles and positions used to recognize the knee-extension exercise

\subsubsection{Thigh-Lift Exercise}

The thigh-lift exercise is done by pulling your knees in toward your chest and elevating your thighs. If knees and hips are represented by $\mathrm{K}$ and $\mathrm{Hi}$, respectively, the thigh-lift exercise is recognized by using the thigh joint angle ?Th derived from a straight line connecting $\mathrm{Hi}$ and $\mathrm{K}$ and a perpendicular line, and by the height of the knees $\mathrm{yK}$. The joint angles and joint positions used to recognize the thigh-lift exercise are shown in Figure 8.

Changes in the thigh joint angle and knee joint position when doing the thigh-lift exercise are illustrated in Figure 9. One can see that the value of the thigh joint angle decreases as the knee position is elevated when doing the exercise. The system recognizes the thigh-lift exercise by focusing on the changing thigh joint angle ?Th and knee joint height $\mathrm{yK}$ of the exerciser, and determines that the exercise is being performed when these values satisfy the relevant thresholds.

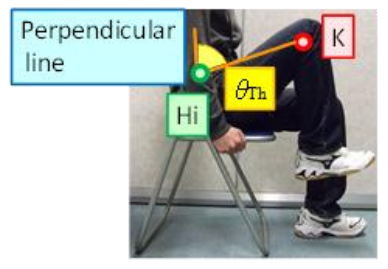

(a) Joint angles

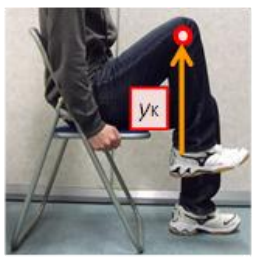

(b) Joint positions

Figure 8: Joint angles and positions used in the knee-lift exercise

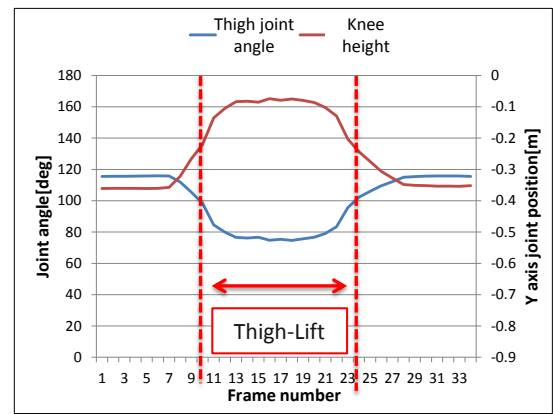

Figure 9: Changing joint angles and positions during the thigh-lift exercise. 


\subsubsection{Leg-Open Exercise}

The leg-open exercise is done by opening the hip joint by moving one leg laterally to the side. Where $\mathrm{K}=$ knees, $\mathrm{m}_{-} \mathrm{Hi}=$ hip on the leg-open side, and $\mathrm{s}_{-} \mathrm{Hi}=$ the hip on the opposite side, the leg-open exercise is recognized by using the hip joint angle $\theta_{c}$ created by straight lines connecting $\mathrm{m}_{-} \mathrm{Hi}$ and $\mathrm{K}$ and connecting $\mathrm{m}_{-} \mathrm{Hi}$ and $\mathrm{s}_{-} \mathrm{Hi}$, and position $x_{\mathrm{K}}$ in the lateral direction the knee is moving. The joint angles and joint positions used to recognize the leg-open exercise are shown in Figure 10.

Changes in the hip joint angle and knee joint position when doing the leg-open exercise on the right foot are shown in Figure 11. One can see in the figure that the value of the hip joint angle increases while the position of the right foot moves laterally along the Kinect $X$ axis, that is, to the right from the exerciser's perspective. The system recognizes the leg-open exercise by focusing on the changing hip joint angle $\theta_{c}$ and knee joint position $x_{k}$ of the exerciser, and determines that the exercise is being performed when these values satisfy the relevant thresholds.

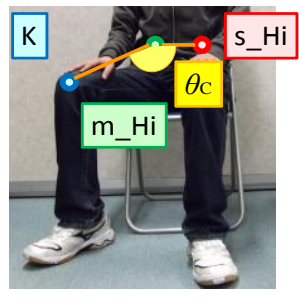

(a) Joint angles

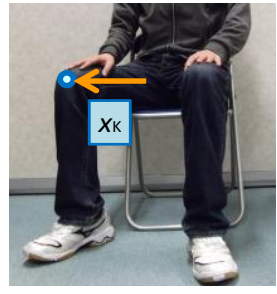

(b) Joint positions

Figure 10: Joint angles and positions used to recognize the leg-open exercise

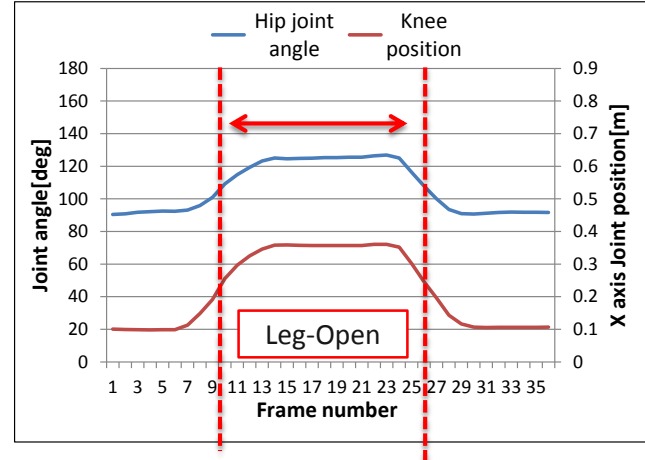

Figure 11: Changing joint angles and positions for recognizing the leg-open exercise for the right foot

A summary of the joint angles and joint positions used to identify five exercises is presented in Table 2.

Table 2: Exercise joint angle and joint position summary

\begin{tabular}{|l|l|l|}
\hline Exercise & Joint angle & Joint position \\
\hline Toe-lift & Ankle joint & Toe height \\
\hline Heel-lift & Ankle joint & Ankle height \\
\hline $\begin{array}{l}\text { Knee- } \\
\text { extension }\end{array}$ & Knee joint & Ankle height \\
\hline Thigh-lift & Thigh joint & Knee height \\
\hline Leg-open & Hip joint & Knee horizontal position \\
\hline
\end{tabular}

\subsection{Evaluating Exercises}

Each of the exercises was evaluated based on the joint angles and joint positions of the exercisers. Here we introduce two thresholds, $\theta_{1}$ and $\theta_{2}$, to subdivide the joint angle $\theta$ of the exerciser. In other words, the two thresholds are used to divide joint angle $\theta$ of the exerciser into three regions, which are assigned evaluations 0,1 , and 2 .

$$
\begin{array}{lc}
\text { Joint angle evaluation 0: } & \theta<\theta_{1} \\
\text { Joint angle evaluation 1: } & \theta_{1} \leq \theta<\theta_{2} \\
\text { Joint angle evaluation 2: } & \theta_{2} \leq \theta
\end{array}
$$


Next, we introduce two thresholds, $w_{1}$ and $w_{2}$, with respect to joint position $w(w=x, y, z)$ of the exercise being done by the exerciser for the rectangular coordinate system $(x, y, z)$. The two thresholds are used to divide joint position $w$ of the exerciser into three regions, which are assigned evaluations 0,1 , and 2 .

$$
\begin{array}{ll}
\text { Joint position evaluation } 0: & w<w_{1} \\
\text { Joint position evaluation 1: } & w_{1} \leq w<w_{2} \\
\text { Joint position evaluation 2: } & w_{2} \leq w
\end{array}
$$

Based on the two thresholds for these two criteria-joint angle and joint position-an exerciser's data can be divided into nine regions. These nine regions are evaluated according to a four-value scale as shown in Table 3, so an exercise can be evaluated as excellent, good, fair, or poor. Referring to the table, one can see that if the user gets "2" for both joint angle and joint position, the exercise is evaluated "excellent." If a user gets a "1" and a "2" for the two criteria, the exercise is evaluated "good." If a user gets "1" for both joint angle and joint position, the exercise will be evaluated as "fair," and if a user gets "0" for either joint angle or joint position, the evaluation for the exercise will be "poor."

Table 3: Exercise joint angle and joint position summary.

\begin{tabular}{|c|c|c|c|}
\hline Joint angle & Evaluation "0" & Evaluation “1" & Evaluation " 2 " \\
\hline Evaluation "0" & poor & poor & poor \\
\hline Evaluation “1" & poor & fair & good \\
\hline Evaluation "2" & poor & good & excellent \\
\hline
\end{tabular}

\section{Implementing a Prototype System}

A prototype system was implemented using Visual C++ 2008, Open CV, and Open NI. The PC used in this work had an Intel(R) Core(TM) i5-2400 CPU with 4 GB of memory. The processing speed was more than adequate to run the prototype implementation without any delays or problems. Figure 12 is a photo of the system in operation. One will observe in the photo that the system is set up toward the back of the room on the right, and the exercise situation is displayed on the screen to the left. The Kinect sensor is deployed directly in front of the screen to assist the user in the foreground do leg exercises.

Figure 13 shows typical screenshots of support system output. The screen on the left is the RGB image of the exerciser with superimposed muscles used by the exerciser. The screen at the upper right provides an example of how each exercise should be done, and the screen at the lower right illustrates the beneficial effects of each exercise. In addition, there is a textual assessment of the effects of each exercise in the lower left hand corner of the screen.

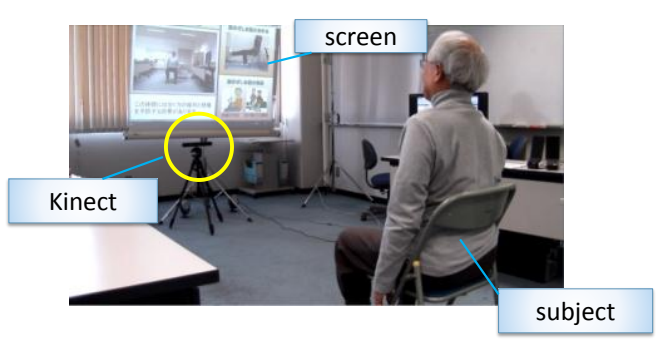

Figure 12: Prototype system in operation

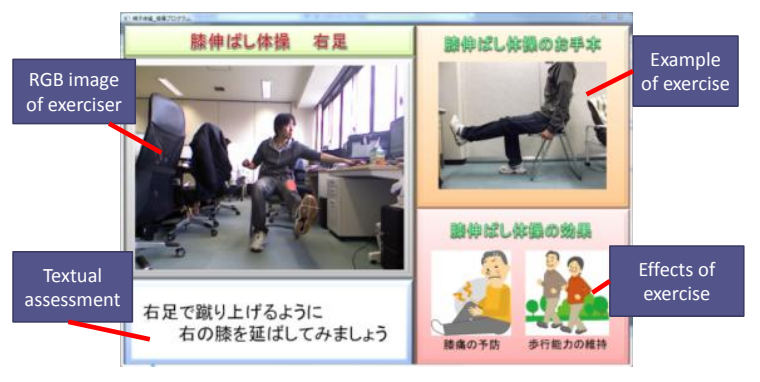

Figure 13: Chair exercise support system output screen. 


\section{Prototype System Trials and Considerations}

To verify the effectiveness of the prototype system, we conducted trials to assess the recognition accuracy of the chair exercise system and also to gauge the response of trial subjects. Figure 14 shows the experimental setup. The Kinect sensor is installed at a height of $72 \mathrm{~cm}$ above the floor, while the subject sits on a folding chair (the seat is $40 \mathrm{~cm}$ above the floor) positioned 2.5 meters from the Kinect. To enhance the recognition accuracy, the subjects kept their shoes on during the trials and loose trouser legs were secured with rubber bands around the ankles. This not only improved the toe position estimation accuracy, it also reduced the adverse effects of loose trouser legs on the Kinect ankle joint position estimation and improved the ankle joint estimation accuracy.

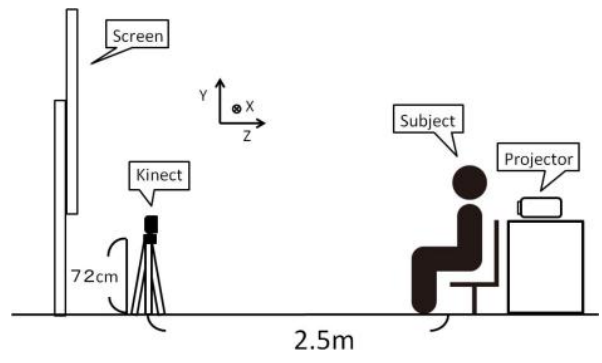

Figure 14: Trial setup

\subsection{Chair Exercise Recognition Accuracy Trials}

These trials were conducted to assess the recognition accuracy of lower limb chair exercises intended for seniors, and to verify the effectiveness of the proposed exercise recognition method. Working with seven subjects ranging in age from latter 70s to early 80 s, we asked the subjects to perform five repetitions of each of the five lower limb exercises on the left and the right. As the subjects did the exercises, we recorded the number of times they executed the exercises correctly, the changes that occurred in the joint angles and positions as they did the exercises. The exercises were done in the following sequence (1) toe-lift, (2) heel-lift, (3) knee-extension, (4) thigh-lift, and (5) leg-open, starting on the right foot and with a three-beat interval between each exercise.

Average recognition rates for the chair exercises are shown in Figure 15. If the rate of correct execution is the number of times an exercise is done correctly divided by the number of times the exercise was attempted, then the average recognition rate is the total correct rate of each exercise divided by the number of exercisers

Average recognition ratefor eachexercise $(\%)$

$$
=\frac{\text { Total correct rate of each exercise }}{\text { Number of subjects }} \times 100
$$

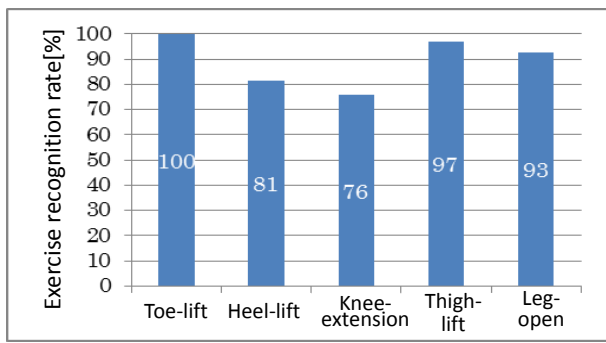

Figure 15: Average recognition rate of the chair exercises 
As one can see in Figure 15, the recognition rate for three of the exercises-toe-lift, thigh-lift, and legopen-is over $90 \%$, and the average recognition accuracy for all five exercises is $89 \%$. The accuracy of the prototype chair exercise system is thus more than sufficient to support at least three of the chair exercises.

Yet, the recognition accuracies for the heel-lift and knee-extension exercises were only $81 \%$ and $76 \%$, respectively. The low recognition rate for the heel-lift exercise is attributed to inadequate tracking of the foot region by Kinect, which results in low accuracy for the foot joint position. One thing we could do to improve the accuracy would be to have seniors warm up with a stepping exercise before starting the chair exercise; this would improve the foot joint position accuracy captured by Kinect. The poor recognition rate for the knee-extension exercise is attributed to the fact that the knee joint is obscured when the subject's foot comes up, which causes Kinect's estimated joint position to be off. We are now reconsidering the exercises to come up with a solution to these problems.

\subsection{Threshold Trials of Thigh-lift}

Figure 16 shows changes in the thigh joint angles and knee heights for the seven subjects doing right thigh-lift exercises. As one can see in the figure, four colors show four evaluation levels.

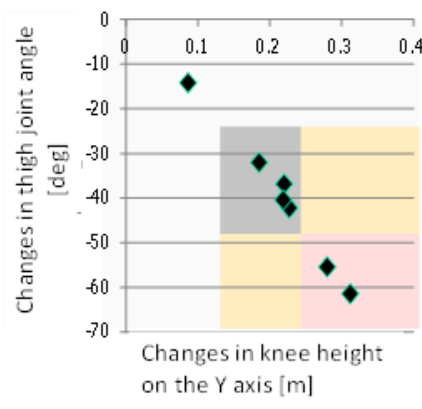

(a) Threshold setting for healthy seniors

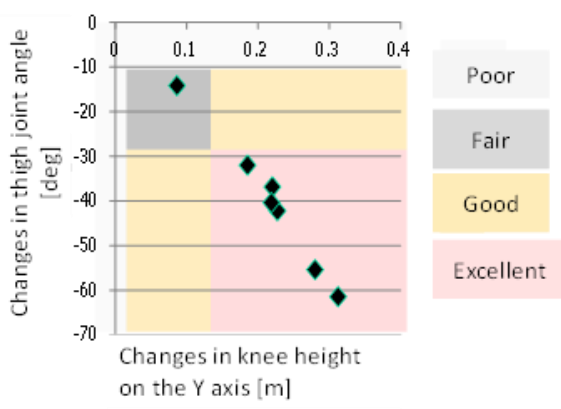

(b) Threshold setting for seniors with lower limb disabilities

Figure 16: Changes during the thigh-lift exercise

In Figure 16, the thigh joint angle of the subject with the greatest amount of change in the thigh joint angle is reduced by an average of 61 degrees, and the knee height of the subject is elevated by $31 \mathrm{~cm}$. These values are practically the same as those for a young person doing the same thigh-lift exercise, which reveals that there are at least some seniors close to 80 years old who perform the same exercises as young people. This also illustrates the importance of identifying exercise evaluation thresholds for senior citizens who are in good physical condition that will help them sustain their physical functions. Figure 16(a) shows threshold settings that would be appropriate for healthy physically fit seniors.

Where $\theta=-\theta_{\text {Th }}$ with respect to thigh joint angle $\theta_{\text {Th, }}$ joint angle evaluations for two thresholds $\theta_{1}$ and $\theta_{2}$, where $\theta_{1}<\theta_{2}$, are set according to conditions (1), (2), and (3) of Section 3.E. Similarly, joint position evaluations for two thresholds $y_{1}$ and $y_{2}$ (where $y_{1}<y_{2}$ ) for the knee height $w=y_{k}$ are set according to conditions (4), (5) and (6). So, for example, the thigh-lift exercise thresholds for seniors in good physical condition shown in Figure 16(a) are set as follows:

$$
\theta_{1}=24 \mathrm{deg}, \theta_{2}=48 \mathrm{deg}, y_{1}=13 \mathrm{~cm}, y_{2}=24 \mathrm{~cm}
$$

Now assuming that the subject with the least amount of change in the thigh joint angle is Subject $E$, the thigh joint angle for this subject performing the exercise is reduced by an average of only 14 degrees while the knee height of the subject is elevated by $9 \mathrm{~cm}$. The changes in joint angle and joint position when Subject E performs the thigh-lift exercises are shown in Figure 17. The ability to set the 
Toshiya Watanabe, Naohiro Ohtsuka, Susumu Shibusawa, Masaru Kamada and Tatsuhiro Yonekura; Design and Development of Lower Limb Chair Exercise Support System with Depth Sensor, Transactions on Networks and Communications, Volume 3 No 4, August (2015); pp: 30-44

thresholds at more modest levels for this elderly individual would provide easier exercises that this user could accomplish. Figure 16(b) illustrates the threshold settings that might be appropriate for this less fit individual.

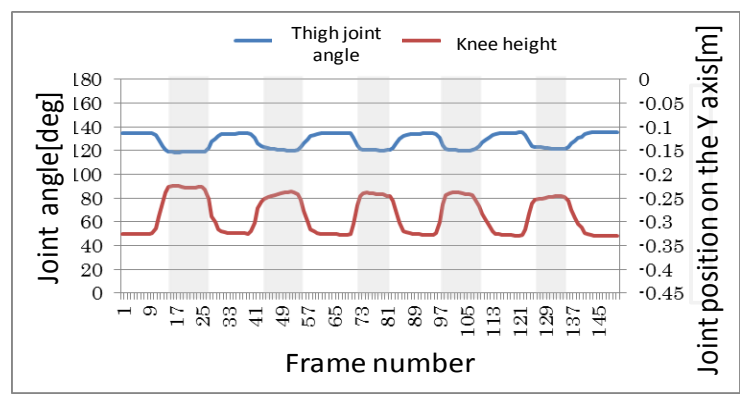

Figure 17: Changes in joint angle and joint position when Subject E does thigh-lift exercises.

The thigh-lift exercise thresholds for seniors requiring the easier exercises shown in Figure 16(b) are set as follows:

$$
\theta_{1}=11 \mathrm{deg}, \theta_{2}=28 \mathrm{deg}, y_{1}=2 \mathrm{~cm}, y_{2}=13 \mathrm{~cm}
$$

Currently, these thresholds values must be set by the system developer.

\subsection{Chair Exercise Support System Evaluation Trials}

To assess the effectiveness of the prototype chair exercise system's support functions, we asked the subjects to fill out a questionnaire evaluating the system. Five male subjects ranging in age from 50 to 65 worked out on the exercise support system, then evaluated the system by responding to a series of questions on a scale of one to five. For the purposes of this trial, the exercises were done in the following sequence (1) toe-lift, (2) heel-lift, (3) knee-extension, (4) thigh-lift, and (5) leg-open, starting on the right foot and each exercise was sustained for 5 seconds.

Appendix A.1 shows the questions that were asked on the questionnaire after using the system. The average points received for each item on the questionnaire are shown in Figure 18. The subjects were asked to respond to each item on a scale from one (strongly disagree) to five (strongly agree). The average for all items on the questionnaire was 4.3 points. The average score was 4.8 on both Question 1 (Were you able to understand the five types of chair exercises provided by the system?) and on Question 10 (Do you think there is no need to learn a lot preliminary stuff before using the system?). It is clear from these responses that the chair exercises supported by the prototype system are fairly easy to understand and perform.

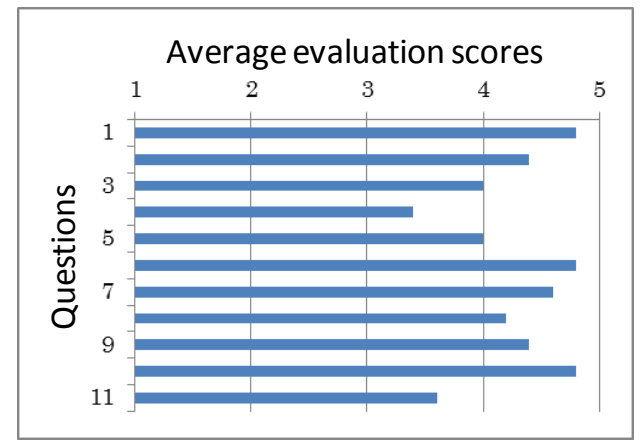

Figure 18: Evaluation trial results 
In response to Question 4 (Were you aware of which muscles are used when doing the exercises?), the average score was 3.4, which was fairly low. We attribute this low rating to the fact that the subjects were so engrossed in doing the chair exercises correctly that they did not have time to think about the muscular effects. In order to promote exercises that encourage users to use their muscles correctly, we would have to provide support functions and exercise programs that are designed to draw attention to the muscles and how they are used.

The questionnaire also provided space for the subjects to offer their own free assessments of the system, and several of the subjects made the observation that "an exercise program you could use often or everyday would be especially useful." Clearly, this would require a number of different exercise programs tailored for different fitness objectives. For example, by offering a number of different programs to help strengthen not just the lower limbs but other parts of the body, this would go a long way toward stimulating continuity and spontaneity of users. By working together with physical therapists and other healthcare professionals, it should be possible to create exercise programs that are even more effective than the prototype system we present here.

Several of the subjects also commented that "compared to how I lived my life in the past, I want to figure out a way to stay physically active and healthy." We found that seniors derive a sense of security and joy when they are physically fit, and this is clearly linked to their persistence and motivation to continue exercising. An additional display function enabling users to compare their own past data with their current exercise data would help motivate users stick with the exercise program.

Finally, establishing ties with other seniors through exercise is extremely important to sustain interest and motivate users to continue exercising. Working out or exercising as a group enables seniors to express themselves and project their individuality. The exercise support system can thus be seen as a basic social mechanism for getting the elderly out of their homes and rooms and bringing them together for a social activity.

\section{Conclusions}

In this study we designed and built a prototype lower limb chair exercise support system using a depth sensor, and evaluated the functionality and effectiveness of the system through trials on a number of elderly subjects. The system provides examples of how each exercise should be done using voice instructions and model images, while also showing the muscles used superimposed onto real-time images of the exerciser performing the exercises. The system also provides exercise assessment results and advice by voice and text.

To evaluate the prototype system, we tested its recognition accuracy on elderly subjects ranging in age from late 70 s to early $80 \mathrm{~s}$. The overall recognition accuracy for all five chair exercises was $89 \%$, but the recognition rate was lower for two of the exercises-the heel-lift exercise and the knee extension exercise-due to relatively poor Kinect estimates of joint positions. To get feedback from actual users, we also conducted an assessment trial of the system on subjects ranging in age from 50 to 65 . An overall score of 4.3 was obtained on a five-point scale, but a couple of the questions drew lower scores. On the free comments section of the questionnaire, subjects mentioned that they "would like an exercise program they could do every day" and are looking "for a way to stay physically active and healthy."

Building on the work done so far, there are several aspects of the system we hope to enhance. First, the exercise recognition accuracy of the system could be improved. We also identified a number of additional capabilities that would enhance spontaneity and encourage seniors to persist with the exercise routine: a game element that encourages users to exercise, additional exercise programs 
Toshiya Watanabe, Naohiro Ohtsuka, Susumu Shibusawa, Masaru Kamada and Tatsuhiro Yonekura; Design and Development of Lower Limb Chair Exercise Support System with Depth Sensor, Transactions on Networks and Communications, Volume 3 No 4, August (2015); pp: 30-44

tailored for other purposes that strengthen other muscles, and a way to visualize a user's current exercise capabilities compared with exercise data from the past. The system should also be adjustable so the exercise administrator can easily adapt the system to the fitness level of the exercisers.

\section{ACKNOWLEDGEMENTS}

The authors wish to express their appreciation to the staff of the Hitachi City Social Services Division for the Elderly, and the residents at the Kashima Retirement Home and Sukegawa Community Center.

\section{REFERENCES}

[1] Cabinet Office, 2015 Annual Report on the Aging Society, http://www8.cao.go.jp/kourei/whitepaper/w-2015/zenbun/ index.html, Accessed June 18, 2015.

[2] Ministry of Health, Labour and Welfare, Manual of Long-Term Care, http://www.mhlw.go.jp/topics/2009/ 05/tp0501-1.html, Accessed June 18, 2015.

[3] Matsukuma, H., Fujioka, S., Nakajima, A., Kaneko, K., Kajiwara, J., Hayashida, K., and Hattori, F., Research and development of serious games to support stand-up rehabilitation exercises, Journal of Information Processing, 53(3), 1041-1049, March 2012.

[4] Hashimoto, Y., Munesawa, T., Mitsuto, R., Tanabe, K., Masumoto, K., Morimoto, R., Sawada, K., and So, B. C., Internet technology in cognitive rehabilitation: review and pactice, Trans. of the Institute of Electronics, Information and Communication Engineers, J95-D(5), 10911099, May 2012.

[5] Cognitive Rehabilitation Anywhere, http://reha.heteml.jp , Accessed June 18, 2015.

[6] Mentiplay, B. F., Clark, R. A., Mullins, A., Bryant, A. L., Bartold, S., and Paterson, K., Reliability and validity of the Microsoft Kinect for evaluating static foot posture, Journal of Foot and Ankle Research 2013， 6-14， 2013.

[7] Metcalf, C. D., Robinson, R., Malpass, A. J., Bogle, T. P., Dell, T. A., Harris, C. and Demain, S. $\mathrm{H}$., Markerless motion capture and measurement of hand kinematics: validation and application to home-based upper limb rehabilitation, IEEE Trans. on Biomedical Eng., 60(8), 2184-2192, Aug. 2013.

[8] Obdrzalek, S., Kurillo, G., Ofli, F., Bajcsy, R., Seto, E., Jimison, H., and Pavel, M., Accuracy and robustness of Kinect pose estimation in the context of coaching of elderly population, The 34th Annual Int. Conf. of the IEEE Engineering in Medicine and Biology Society EMBC'12, 1188-1193, 2012.

[9] Erazo, O., Pino, J. A., Pino, R., Asenjo, A., Fernández, C., and Asenjo, A., Magic mirror for neurorehabilitation of people with upper limb dysfunction using Kinect, The 47th Hawaii Int. Conf. on System Sciences HICSS, 2607-2615, Jan. 2014. 
[10] Webster, D. and Celik, O., Systematic review of Kinect applications in elderly care and stroke rehabilitation, Journal of NeuroEngineering and Rehabilitation, 11(108), 2014.

[11] Watanabe, T., Ohtsuka, N., Shibusawa, S., Kamada, M., and Yonekura, T., Design of lower limb chair exercise support system with depth sensor, The 11th IEEE Int. Conf. on Ubiquitous Intelligence and Computing, 104-111, Dec. 2014.

[12] Watanabe, T., Ohtsuka, N., Shibusawa, S., Kamada, M., and Yonekura, T., Motion detection and evaluation of chair exercise support system with depth image sensor, Int. Workshop on Future Trends in Computing System Technologies and Applications, 800-807, Dec. 2014.

[13] Tolentino, G. P., Battaglini, C., Pereira, A. C. V., Oliveria, R. J., and Paula, M. G. M., Usability of serious games for health, The Third Int. Conf. on Games and Virtual Worlds for Serious Applications VS-GAMES, 172-175, May 2011.

[14] Moya, S., Grau, S., Tost, D., Campeny, R., and Ruiz, M., Animation of 3D avatars for rehabilitation of the upper limbs, The Third Int. Conf. on Games and Virtual Worlds for Serious Applications VS-GAMES, 168-171, May 2011.

[15] Bruckheimer, A. D., Hounsell, M. S., and Soares, A. V., Dance2Rehab3D: A 3D virtual rehabilitation game, The 14th Symp. on Virtual and Augmented Reality, 182-190, May 2012.

[16] Asakura, R., Miyasaka, J., Kondo, K., Nakamura, Y., Akita, J., Toda, M., and Sakurazawa, S., Design of a rehabilitation supporting system integrating myoelectric sensing and imagebased pose sensing, Trans. of the Institute of Electronics, Information and Communication Engineers, J97-D(1), 50-61, Jan. 2014.

[17] Pei, Y., Kim, Y., Obinata, G., and Hase, K., Design of motion trajectory and external force on foot based on musculo-skeletal model in robot-assisted lower limb rehabilitation, Trans. of the Japan Society of Mechanical Engineers, Series C, 77(781), 236-250, Sept. 2011.

[18] Fasola, J. and Mataric, M. J., Using socially assistive human-robot interaction to motivate physical exercise for older adults, Proc. of the IEEE, 100(8), 2512-2526, Aug. 2012.

[19] Igarashi, N., Suzuki, K., Kawamoto, and H., Sankai, Y., A Wearable light-emitting sensor suit for supporting the lower-limb motion perception, Journal of Information Processing, 53(4), 1360-1371, April 2012.

[20] Reflexion Health, http://reflexionhealth.com/ , Accessed July 22, 2015.

[21] Virtualrehab, http://www.virtualrehab.info/ , Accessed July 22, 2015.

[22] Seeme, http://www.virtual-realityrehabilitation.com/products/ seeme/what-is-seeme, Accessed July 22, 2015.

[23] Jintronix, http://www.jintronix.com/ , Accessed July 22, 2015. 
Toshiya Watanabe, Naohiro Ohtsuka, Susumu Shibusawa, Masaru Kamada and Tatsuhiro Yonekura; Design and Development of Lower Limb Chair Exercise Support System with Depth Sensor, Transactions on Networks and Communications, Volume 3 No 4, August (2015); pp: 30-44

[24] Patent WO 2013090554 A1, Method and system for evaluating a patient during a rehabilitation exercise, Publication date June 20, 2013. Also, Patent US 20140371633 A1, Publication date Dec. 18, 2014.

[25] Tao, G., Archambault, P., and Levin, M. F., Evaluation of a virtual reality rehabilitation system for upper limb hemiparesis, Int. Conf. on Virtual Rehabilitation ICVR, pp.164-165, Aug. 2013.

[26] Norouzi-Gheidari, N., Levin, M. F., Fung, J., and Archambault, P., A research protocol exploring the use of haptic forces for stroke rehabilitation, Int. Conf. on Virtual Rehabilitation ICVR, pp.220-221, Aug. 2013. 
APPENDIX

\section{A.1 Items on the Chair Exercise Support System Evaluation Questionnaire}

Table A.1: Questionnaire Items.

\begin{tabular}{|c|l|}
\hline 1 & Were you able to understand the five types of chair exercises provided by the system? \\
\hline 2 & By continuing with the exercises, to you think this would have beneficial effect on preventative care? \\
\hline 3 & Did the muscle display function help you understand how muscles are trained and strengthened? \\
\hline 4 & Were you aware of which muscles are used when doing the exercises? \\
\hline 5 & Were displays of illustrated effects of exercise easy to understand? \\
\hline 6 & Was the 4-grade exercise scale useful for measuring exercise progress? \\
\hline 7 & Was the real-time color exercise evaluation display useful for measuring exercise progress? \\
\hline 8 & Did you think the system was easy to use? \\
\hline 9 & Do you think the typical user could quickly figure out how to use this system? \\
\hline 10 & Do you think there is no need to learn a lot of preliminary stuff before using the system? \\
\hline 11 & Would you be interested in using this system again? \\
\hline
\end{tabular}

